\title{
Systematic computational SAR analysis
}

\section{J Bajorath}

\author{
Address: Department of Life Science Informatics, B-IT, LIMES Program Unit Chemical Biology and Medicinal Chemistry, Rheinische Friedrich- \\ Wilhelms-Universtität Bonn, Dahlmannstr. 2, D-53113 Bonn, Germany \\ from 4th German Conference on Chemoinformatics \\ Goslar, Germany. 9-II November 2008 \\ Published: 5 June 2009 \\ Chemistry Central Journal 2009, 3(Suppl I):O2 doi:I0.II86/I752-I53X-3-SI-O2
}

This abstract is available from: http://www.journal.chemistrycentral.com/content/3/SI/O2

(C) 2009 Bajorath; licensee BioMed Central Ltd.

In medicinal chemistry, the exploration of structure-activity relationships (SARs) plays a fundamental role in hitto-lead projects and lead optimization programs. Typically, SARs are explored on a case-by-case basis and comparative or large-scale SAR analyses are rarely reported.

We aim at a systematic computational exploration of global and local SAR characteristics of compound data sets. Only few attempts have been made thus far to qualitatively evaluate global SAR features, for example, through the introduction of Structure-Activity Similarity Maps [1] or 2D/3D Similarity Correlation Graphs [2]. In addition, global and local SAR characteristics have also been quantitatively assessed by means of the SAR [3] and SALI [4] function, respectively.

Here compound-based SARI scoring and annotated molecular network representations are introduced that make it possible to systematically relate global and local SAR features to each other and identify key compounds that are SAR determinants.

\section{References}

I. Shanmugasundaram V, Maggiora GM: 222nd ACS Natl Meeting $200 \mathrm{I}$. Abstract no.77.

2. Peltason L, Bajorath J: Chem Biol 2007, 14:489-497.

3. Peltason L, Bajorath J: J Med Chem 2007, 50:557I-5578.

4. Guha R, Van Drie JH: J Chem Inf Model 2008, 48:646-658. 